\title{
Oncogene SKI
}

National Cancer Institute

\section{Source}

National Cancer Institute. Oncogene SKI. NCI Thesaurus. Code C18449.

This gene is involved in transcriptional regulation and is involved in cellular signal transduction. 